\title{
Luminous Red Galaxies at Redshifts, $\mathrm{z}=0.4-0.5$
}

\author{
Sara R. Heap ${ }^{1}$ and Don Lindler ${ }^{1,2}$ \\ ${ }^{1}$ NASA's Goddard Space Flight Center, Greenbelt MD 20771 USA \\ email: Sally.Heap@NASA.gov \\ ${ }^{2}$ Sigma Space, Inc., Lanham MD, USA \\ email: lindler@milkyway.gsfc.nasa.gov
}

Abstract. We describe and analyze the color dispersion of LRG's at redshifts, $\mathrm{z}=0.4-0.5$.

Keywords. luminous red galaxies, spectra, stellar populations, Sloan Digital Sky Survey

Luminous red galaxies (LRG's) at redshift, $\mathrm{z}=0.4-0.5$, are a bridge to high-redshift LRG's in both a scientific and technical sense. At $\mathrm{z}=0.45$, we are seeing LRG's when they were 4.6 Gyr younger, so we can start to trace how local LRG's came to be. At this moderate redshift, the mid-ultraviolet is shifted into the optical, so restframe spectra of these LRG's from the Sloan Digital Sky Survey (SDSS) span $\sim 2500-6700 \AA$. This spectral region includes all the Lick indices, which can be used to estimate the ages and metallicities of these galaxies. In principle, we can then calibrate the restframe UV in terms of age and metallicity given a knowledge of their properties gained from the optical spectrum.

We obtained SDSS spectra of about 25,000 spectra of LRG's at $z=0.4-0.5$, of which 21,064 were usuable. We scaled the restframe fluxes to a single distance $(\mathrm{z}=0.45)$, measured their restframe magnitudes, $u$ (3000-3500 $\AA), b(4200-4800 \AA)$, and $y$ (5300-6100 $\AA$ ), and stacked the spectra according to their $u$ - $b$ colors and $\mathrm{M}_{y}$ absolute magnitudes. Figure 1 shows a color-color plot of the 21064 LRG's with model galaxy colors from BaSTI overplotted. The plot suggests that the galaxies must have different ages and/or metallicities. However, the Lick indices imply a near-uniform age, $\sim 7$ Gyr, and metallicity, $[\mathrm{Fe} / \mathrm{H}] \sim 0$ (results from the program, EZ_ages, by Graves \& Schiavon 2008).

Figure 2 compares the UV-blue portion of the spectrum in the bluest, typical, and reddest galaxies of the same absolute magnitude. The spectral absorption features are similar in detail, except that the magnesium resonance lines are less deep in the bluer galaxies despite the fact that the MgII $\lambda 2800$ resonance doublet must be saturated. It suggests that the UV lines lie on a "pedestal" of light contributed by a UV-bright stellar population.

The question then becomes whether the UV-bright stellar population is composed of young hot stars (Yi et al. 2005) or metal-poor stars that are the remnant of the original star-forming events (Maraston et al. 2009). We have used BaSTI isochrones combined with model spectra from Castelli \& Kurucz (2003) and MARCS (2008) to compute model spectra of both stellar populations. We find an equally good/poor fit for these two cases. However, the strength of the unidentified absorption feature at $\lambda 2881$ (associated with cool stars) in the spectrum of the bluest favors the latter interpretation. 


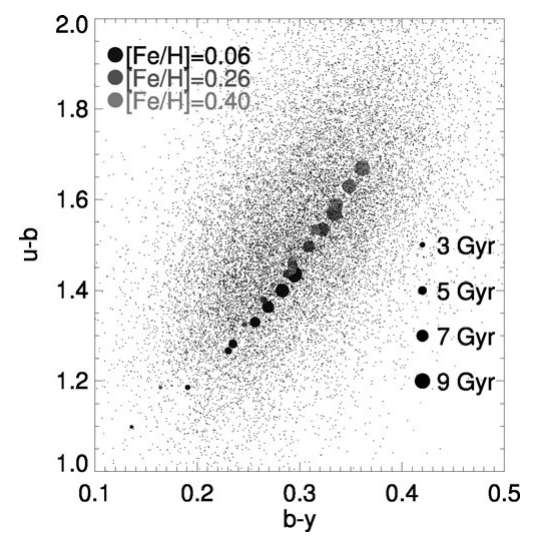

Figure 1. Color-color plot of LRG's.

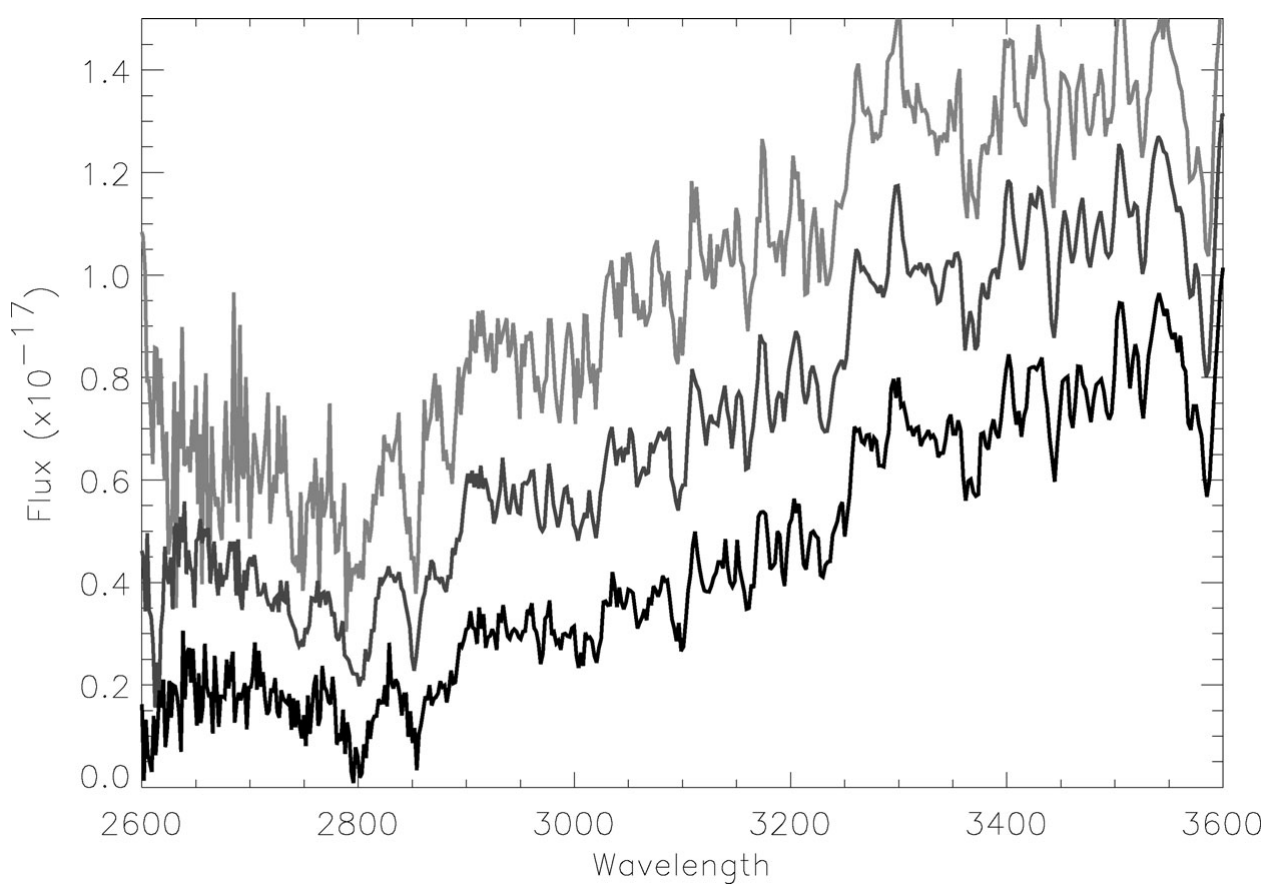

Figure 2. UV-blue segments of the bluest, typical, and reddest LRG's (top to bottom) of the same absolute visual magnitude, i.e. the three spectra converge at $5500 \AA$.

\section{References}

BaSTI 2008, http://albione.oa-teramo.inaf.it/

Castelli, F. \& Kurucz, R. 2003, in IAU Symp. No 210, Modelling of Stellar Atmospheres, eds. N. Piskunov et al., poster A20

Graves, G. \& Schiavon, R. 2008, ApJS 177, 446

Maraston, C., et al. 2009, MNRAS (Letters) 394, L107

MARCS 2008, http://marcs.astro.uu.se

Yi, S., et al. 2005, ApJ (Letters) 619, L111 